\title{
The feasibility and usefulness of oximetry measurements in primary care
}

\author{
Kevin Jones, Paul Cassidy, Jeremy Killen, Helen Ellis
}

\begin{abstract}
Aim:

To explore the feasibility and usefulness of oximetry measurements in primary care.

\section{Methods:}

Data collection over six months in 17 volunteer general practices in Gateshead using the Nellcor NPB-40 pulse oximeter and a simple data recording form.

\section{Results:}

229 measurements of oxygen saturation were made with $65 \%$ in the $61-80$ years age group. 30 patients $(13 \%)$ were found to have readings of $90 \%$ or lower; ten were admitted and four referred for outpatient assessment. The readings reassured the doctor and the patient in more than $60 \%$ of cases.
\end{abstract}

\section{Conclusions:}

With minimal training, doctors and nurses made significant use of the oximeters, gaining valuable reassurance in the majority and identifying potentially important hypoxia in the minority. Greater use of pulse oximetry may be a way of making more targeted use of scarce hospital-based specialist services.

\section{Key words:}

Pulse oximetry, COPD, primary care.

\section{Kevin Jones}

Honorary Senior Lecturer in Primary Health Care

Paul Cassidy

GP Teams Practice

Gateshead

\section{Jeremy Killen}

Consultant Chest

Physician, Queen

Elizabeth Hospital,

Gateshead

\section{Helen Ellis}

Respiratory Specialist Nurse, Queen Elizabeth Hospital, Gateshead

Correspondence to:

Dr Kevin Jones

School of Population and

Health Sciences

The University of

Newcastle

Framlington Place

Newcastle NE2 4HH

Tel: +44 (0)191-222-8897 e-mail:

k.p.jones@ncl.ac.uk

Date submitted: 08/10/02

Date Accepted: 15/01/03

Prim Care Resp J 2003; 12(1):4-6

\section{Introduction}

The publishing in 1997 of the British Thoracic Society (BTS) Guidelines on the management of chronic obstructive pulmonary disease (COPD) ${ }^{1}$ greatly increased the focus placed on this common and very distressing disease in both primary and secondary care. Many general practices have recognised that many patients previously labelled as having asthma probably have COPD either instead of or as well as asthma and have sought to alter their services provided to cope with this change.

The provision of spirometry measurements either in house or by open access to hospital respiratory function laboratories was recognised as a priority in COPD care in the community early on, mainly for diagnostic purposes, but the use of other technology has been much slower. One example of other possibilities is the measurement of oxygen saturation by pulse oximetry. This can be helpful in determining which patients with stable COPD might benefit from long term oxygen therapy (LTOT) and also which acutely unwell patients may need hospital admission. Potentially, both in-patient and outpatient referrals can be better targeted by using pulse oximetry and thus make more optimal use of scant specialist resources.

A previous two practice study in London surveyed 114 patients with COPD over 12 months and identified thirteen with saturations of $92 \%$ or below. ${ }^{2}$ Three went on to receive LTOT after hospital assessment. The use of pulse oximeters as a screening tool for hypoxia (using a cut-off point of $92 \%$ ) in 64 patients seen in an emergency department with acute exacerbations of COPD has been shown to have a sensitivity of $100 \%$ and a specificity of $86 \% .^{3}$ Furthermore, the BTS guidelines on the management of community acquired pneumonia in adults concluded that 'pulse oximetry with appropriate training should become increasingly available to GPs for the assessment of severity and oxygen requirements for patients with community-acquired pneumonia and other acute respiratory illnesses'. ${ }^{4}$

This pragmatic study aimed to explore the feasibility and usefulness of oximetry measurements in a larger sample of practices from a significantly more deprived area of the UK.

\section{Methods}

The Gateshead Primary Care Group - now a Primary Care Trust (PCT) - administers 32 practices with 119 GPs and serves a population of approximately 210,000 people in a predominantly deprived urban/inner city environment. The PCT has a respiratory sub-group, which has sought to advance and promote the community care of patients with respiratory problems. Regular meetings have been convened and this project is the first research activity to emerge from this initiative.

All 32 practices were invited by the PCT to participate in a pragmatic study of oximetry measurements in primary care over a six-month period from July to December 2001. Funding was obtained to buy 18 Nellcor NPB-40 oximeters, which were then supplied with brief training to 17 volunteer practices and to the out-of-hours deputising service. It was suggested that suitable patients in whom one could measure oxygen saturation were predominantly those with COPD, but use in other circumstances was left to the individual clinician. Practices were left to decide themselves whether doctors or nurses would be the prime users of the equipment but were advised that readings of $90 \%$ or below should lead acutely to hospital admission and chronically to out-patient 


\section{Not to be reproduced without the permission of the Primary Care Respiratory Journal}

referral. On each occasion that the equipment was used, a very short questionnaire was completed (see Box 1). HE, who also provided encouragement to the practices throughout the study, collected these data from the practice. Simple descriptive statistics only were employed. The Gateshead Local Research Ethics Committee gave ethical approval for the study.

\section{Results}

By the end of the six-month period, data on 229 oximetry measurements had been recorded and collected. An unknown number of patients may have appeared more than once in this dataset. Not all data items were completed in every case, but the percentages quoted relate to the total denominator. One hundred and seven measurements $(47 \%)$ were on females and $121(53 \%)$ on males. Sixteen measurements $(7 \%)$ were on subjects aged under 40 years, with $39(17 \%)$ in the age range 41-60 years, 149 (65\%) 61-80 years and $22(10 \%)$ were on subjects aged over 80 years.

The measurements were recorded as being made in surgeries in 149 instances $(65 \%)$ and in the subjects' homes in $39(17 \%)$. The problem being presented was recorded as acute in 36 cases (15\%), chronic in 50 $(22 \%)$ and acute on chronic in $9(4 \%)$ - although the criteria for the last of these were left to the clinician. The oxygen saturations were in the range $91-100 \%$ in 195 cases $(85 \%), 81-90 \%$ in $25(11 \%)$ and $60-80 \%$ in $5(2 \%)$. Of the 30 recordings of $90 \%$ or less, ten patients were admitted and four referred for outpatient assessment.

In 43 cases $(19 \%)$, the clinician reported that the reading changed the management plan. In 154 cases (67\%), the reading reassured the clinician and in 139 cases $(61 \%)$, the reading reassured the patient.

\section{Discussion}

With minimal training, doctors and nurses made significant use of the oximeters during the study. Furthermore, important reassurance for both clinicians and patients resulted from the measurements and thirty patients were identified with potentially significant hypoxia.

The study was a 'real life', pragmatic piece of research and as such the data are less complete that we hoped for. For instance, despite making the data recording form very brief, not everything was recorded as asked. We have no knowledge of how many times the oximeters could have been used with benefit and were not, nor do we have spirometry measurements to match the oxygen saturations. We also do not know that all the readings were taken with correct technique. Nevertheless, the potential benefits of using pulse oximeters in primary care seem worthwhile. The involvement of the Gateshead PCT in the project enabled funding to be gained fairly easily and good numbers of patients to be recruited in a relatively short period of time. The importance of research of this sort carried out by PCTs is likely to grow as these organisations become more established.

Very little other research on using oximeters in primary care as part of COPD management has been conducted. Roberts et al found similar proportions with hypoxia, but used a higher level of $92 \%$ or less. $^{2}$ They cautiously concluded that oximetry may be beneficial but had two main concerns. Firstly, their two London practices were both suburban and affluent - and thus potentially atypical. Our results confirm the applicability of oximetry measurements in a more pragmatic setting using a wider selection of practices from a predominantly more deprived area of the north-east of England. Secondly, in 1994 when their equipment was bought, each unit cost about $£ 1850$ and this expenditure was thought to be prohibitive for most practices. However, our units only cost $£ 500$ and small, very portable units are now available for around $£ 350$. This fall in price surely makes such equipment well within the grasp of most if not all practices.

Our trigger level for action was set at $90 \%$, not $92 \%$, at the request of $\mathrm{JK}$, acknowledging the pressures already operating on a service short of one consultant. If the higher figure had been used, as has been recommended, a further 17 readings would have required action.

We feel that the measurement of oxygen saturations using pulse oximetry is going to play an increasing role in the primary care assessment of COPD in both the acute and chronic settings, and suggest that PCTs should look to include such measurements in their protocols for COPD. Hopefully in due course, a National Service Framework for obstructive airways disease will emerge to reinforce this recommendation.

\section{Acknowledgments}

We should like to thank Gateshead PCT for funding the purchase of the oximeters used in this study and for help in data processing. Thanks are also due to all those doctors and nurses who come to the meetings of the respiratory sub-group and who collected data for this project.

\section{References}

1. BTS Guidelines for the Management of Chronic Obstructive Pulmonary Disease. Thorax 1997; 52 Supplement 5:1S-28S. 
2. Roberts CM, Franklin J, O'Neill A, Roberts RP, Ide J, Hanley ML, Edwards J. Screening patients in general practice with COPD for long-term domiciliary oxygen requirement using pulse oximetry. Respir Med 1998; 92: 1265-8.
3. Kelly A-M, McAlpine R, Kyle E. How accurate are pulse oximeters in patients with acute exacerbations of chronic obstructive airways disease? Respir Med 2001; 95: 336-340.

4. British Thoracic Society. Guidelines for the Management of Community Acquired Pneumonia in Adults. Thorax 2001; 56: supplement IV.

\section{EXTRAORDINARY GENERAL MEETING}

\section{APRIL 2003, 3PM-4PM, NEW CONNAUGHT ROOMS, GREAT QUEEN STREET, LONDON}

In order to achieve charitable status the Charity Commission requires that the GPIAG charitable objects are altered. We are calling an Extraordinary General Meeting to approve the changes to these objects. These are listed below for your consideration.

\section{THE COMPANIES ACTS 1985 AND 1989 COMPANY LIMITED BY SHARES RESOLUTIONS IN WRITING - GENERAL PRACTICE AIRWAYS GROUP}

To consider and if thought fit, to pass the following resolutions of which resolutions 1 and 2 will be proposed as Special Resolutions and resolution 3 will be proposed as an Ordinary Resolution.

1. That clause 3 of the Memorandum of Association of the Company be amended by the addition of the words "for the public benefit" so that the clause shall read;

The Charity's objects ("the Objects") are to promote interest in, educate and facilitate research for the benefit of the public into all aspects of common respiratory conditions found in Primary Care; to provide an authoritative opinion where required on matters relating to all aspects of common respiratory conditions found in Primary Care; accredit and endorse methodologies, research, products, individuals and bodies after proper consideration; and to provide information for subscribers and others on all common respiratory conditions found in Primary Care.

2. That the memorandum of association and articles of association of the Company be amended as follows;

a) By the deletion of the word "Limited" in the heading to the memorandum of association and in clause 1. of the memorandum of association;

b) By the deletion of clause 5(7) of the memorandum of association

c) by the deletion of article 25 and its replacement with a new article 25 to read as follows;

"The number of Trustees shall be not less than three and not more than five"

d) by the addition of a new sub-clause at article 28 to be numbered article 28(3);

"To seek the assistance of special advisors to inform and advise the Trustees in the discharge of their duties; provide that the trustees shall not be bound to seek or accept such advice"

3. That the minutes shall be written up and circulated to the members by the Secretary.

By order of the Trustees

Registered Number 4298947

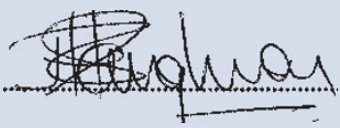

Dr John Haughney

Company Secretary, 14 February 2003

\section{NOTES:}

1.A member entitled to attend and vote at the meeting is entitled to appoint a proxy to attend and, on a poll, vote in his/her place. A proxy need not also be a member. In order to be effective a completed and signed form of proxy in the attached form must reach the Company's registered office not less than 48 hours before the time appointed for the holding of the meeting or any adjournment of it

2.The appointment of a proxy does not prevent a member who so wishes from attending the meeting and voting in person

3. Proxy voting forms can be obtained from the GPIAG secretariat, telephone +44 (0)121 4548219 